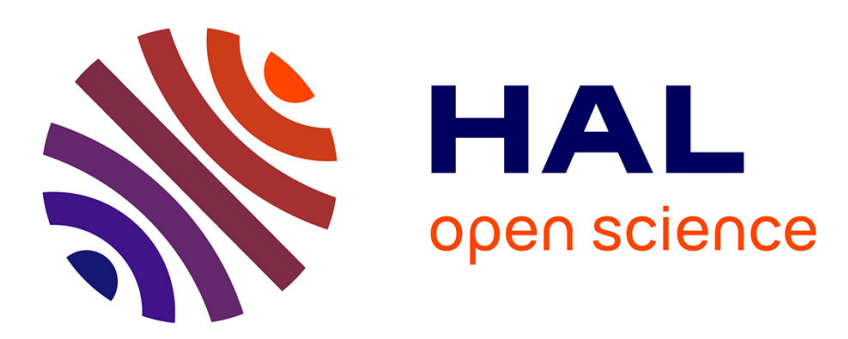

\title{
Evolution du marais de Dol-de-Bretagne au Flandrien ( Ile-et-Vilaine, France)
}

Georgette Delibrias, Marie Thérèse Morzadec-Kerfourn

\section{To cite this version:}

Georgette Delibrias, Marie Thérèse Morzadec-Kerfourn. Evolution du marais de Dol-de-Bretagne au Flandrien ( Ile-et-Vilaine, France). Bulletin de l'Association française pour l'étude du quaternaire, 1975, 12 (2), pp.59 - 70. 10.3406/quate.1975.2083 . hal-03407131

\section{HAL Id: hal-03407131 \\ https://hal.science/hal-03407131}

Submitted on 28 Oct 2021

HAL is a multi-disciplinary open access archive for the deposit and dissemination of scientific research documents, whether they are published or not. The documents may come from teaching and research institutions in France or abroad, or from public or private research centers.
L'archive ouverte pluridisciplinaire HAL, est destinée au dépôt et à la diffusion de documents scientifiques de niveau recherche, publiés ou non, émanant des établissements d'enseignement et de recherche français ou étrangers, des laboratoires publics ou privés. 


\section{Evolution du marais de Dol-de-Bretagne au Flandrien ( Ile-et- Vilaine, France)}

Georgette Delibrias, Marie Thérèse Morzadec-Kerfourn

\section{Citer ce document / Cite this document :}

Delibrias Georgette, Morzadec-Kerfourn Marie Thérèse. Evolution du marais de Dol-de-Bretagne au Flandrien ( Ile-et-Vilaine, France). In: Bulletin de l'Association française pour l'étude du quaternaire, vol. 12, n², 1975. pp. 59-70;

doi : https://doi.org/10.3406/quate.1975.2083

https://www.persee.fr/doc/quate_0004-5500_1975_num_12_2_2083

Fichier pdf généré le 06/11/2020 


\begin{abstract}
The Flandrian deposits filling in the depression corresponding to the present marshes and polderlands around Dol-de-Bretagne are constituted by marine sediments, sands and silts, with intercalations of peat layers. Radio-carbone dates and pollen analytical dates show that these different peaty layers were formed at the end of the Boreal period, towards 8200 B.P., at the end of the Atlantic and the beginning of the Subboreal, betwen 5850 and 5400 B.P. , during the Subboreal betwen 3900 and 3 450 B.P. and during the Subatlantic, from 3000 years B.P. onwards. It seems necessary to suggest slowing down in the transgression (or even regressions) to explain the growing of the peat. A curve of the variations of the sea-level cannot be drawn because of the differential compaction of the sediments under the marshes.
\end{abstract}

\title{
Résumé
}

Le remplissage flandrien de la dépression correspondant aux marais et aux polders de Dol-deBretagne est constitué par des sédiments marins, sables et tangues, dans lesquels sont intercalés des niveaux tourbeux. Des datations radiocarbone et des analyses polliniques montrent que ces diverses lentilles tourbeuses se sont formées à la fin du Boréal, vers 8200 années B.P., à la fin de l'Atlantique — début du Subboréal entre 5850 et 5400 années B.P., au Subboréal entre 3900 et 3450 années B.P. et au Subatlantique à partir de 3000 années B.P. II paraît nécessaire d'invoquer des ralentissements de la transgression (sinon des régressions) pour expliquer leur formation. Une courbe de variations du niveau marin ne peut être tracée à cause du tassement différentiel des sédiments du marais. 


\title{
ÉVOLUTION DU MARAIS \\ DE DOL-DE-BRETAGNE AU FLANDRIEN (Ille-et-Vilaine, France) ${ }^{*}$
}

\author{
par Georgette DELIBRIAS ** \\ et Marie-Thérèse MORZADEC-KERFOURN ***
}

\begin{abstract}
RESUME
Le remplissage flandrien de la dépression correspondant aux marais et aux polders de Dol-de-Bretagne est constitué par des sédiments marins, sables et tangues, dans lesquels sont intercalés des niveaux tourbeux. Des datations radiocarbone et des analyses polliniques montrent que ces diverses lentilles tourbeuses se sont formées à la fin du Boréal, vers 8200 années B.P., à la fin de l'Atlantique - début du Subboréal entre 5850 et 5400 années B.P., au Subboréal entre 3900 et 3450 années B.P. et au Subatlantique à partir de 3000 années B.P. Il paraît nécessaire d'invoquer des ralentissements de la transgression (sinon des régressions) pour expliquer leur formation. Une courbe de variations du niveau marin ne peut être tracée à cause du tassement différentiel des sédiments du marais.
\end{abstract}

\section{ABSTRACT}

The Flandrian deposits filling in the depression corresponding to the present marshes and polderlands around Dol-de-Bretagne are constituted by marine sediments, sands and silts, with intercalations of peat layers. Radio-carbone dates and pollen analytical dates show that these different peaty layers were formed at the end of the Boreal period, towards 8200 B.P., at the end of the Atlantic and the beginning of the Subboreal, betwen 5850 and 5400 B.P., during the Subboreal betwen 3900 and 3450 B.P. and during the Subatlantic, from 3000 years B.P. onwards. It seems necessary to suggest slowing down in the transgression (or even regressions) to explain the growing of the peat. A curve of the variations of the sea-level cannot be drawn because of the differential compaction of the sediments under the marshes.

Afin d'étudier les variations de la ligne de rivage au cours du Flandrien dans la baie du Mont-Saint-Michel, douze sondages ont été réalisés dans la partie sud-ouest du marais de Dol-de-Bretagne. La baie du Mont-SaintMichel est célèbre pour l'importance de l'amplitude de la marée : $13 \mathrm{~m}$ en moyenne, $14,20 \mathrm{~m}$ pendant les marées d'équinoxe. La mer se retire à $5 \mathrm{~km}$ lors des basses mers à l'Ouest de la Baie, mais à l'Est, près du Mont, le recul peut atteindre $15 \mathrm{~km}$.

La tradition populaire rapporte qu'au début de notre ère une importante forêt entourait le MontSaint-Michel. Elle s'appuie sur la présence de troncs d'arbres (les "coirons") sous les sables marins du

(*) Manuscrit déposé le 4 mars 1975.

(**) Centre des Faibles Radioactivités, C.N.R.S., B.P. 1, 91190 - Gif-sur-Yvette.

(***) Institut de Géologie, Université de Rennes, B.P. 25 A, 35031 - Rennes Cedex, et Equipe de Recherche $\mathrm{n}^{\circ} 27$ du C.N.R.S. 
marais de Dol. Selon l'opinion répandue par l'Abbé Manet (1829) cette forêt aurait été détruite par une marée extraordinaire en 709.

Le site du Mont-Saint-Michel est également apparu très tôt dans la littérature géologique concernant les variations de la ligne de rivage au Quaternaire (S. Sirodot 1878, 1891 ; P. Lebesconte, 1898 ; R. Musset, 1922 ; G. Dubois, 1924 . . .).

Situation géographique du marais de Dol-de-Bretagne (fig. 1)

Le marais de Dol est séparé de la baie du MontSaint-Michel par une digue, longue de $20 \mathrm{~km}$, établie en partie, entre le Château Richeux et la Chapelle Sainte-Anne, sur le cordon littoral flandrien. Le schorre occupe actuellement une frange étroite en bordure du marais le long du cordon littoral ; à l'Est par contre, entre la Chapelle Sainte-Anne et le Couesnon, le long de la digue, il est beaucoup plus étendu et progresse encore.

Protégé par la digue, le marais de Dol est formé par la juxtaposition de trois unités morphologiques qui s'étendent vers le Sud jusqu'au pied de l'abrupt, considéré comme une ancienne falaise :

- un marais tourbeux, ou marais noir au Sud-Ouest (Châteauneuf - Saint-Coulban, Grande Bruère, Pont Labat) ;
- un marais blanc, polder naturel, formé de sables marins intertidaux; ce marais est parcouru par d'anciens cordons littoraux (Grand Sillon, Sillon de la Fresnais) plus ou moins parallèles au cordon littoral actuel ;

- un polder, à l'Est près de l'embouchure du Couesnon, gagné sur la mer entre 1851 et 1934.

\section{Localisation des sondages (fig. 2)}

Les premiers sondages réalisés dans le marais de Dol en 1876, sous la direction de l'Ingénieur Mazellier, avant la construction de la voie ferrée Dol-Lamballe, avaient révélé une stratigraphie comparable à celle des marais flamands et picards (G. Dubois, 1924). Cette stratigraphie a été largement confirmée par la nouvelle campagne de sondages entreprise par le Centre des Faibles radio-activités pour une étude des variations du niveau marin holocène.

Nous avons retenu, avec P.R. Giot, un certain nombre de points d'implantation susceptibles de fournir la succession quaternaire la plus complète, en nous aidant des résultats obtenus par Mazellier et des travaux récents du laboratoire de Géomorphologie de Dinard (F. Verger, 1968 ; J.Y. Desdoigts, 1970). Les prélèvements ont été effectués par carottage continu, sous gaine plastique au cours du printemps 1969.

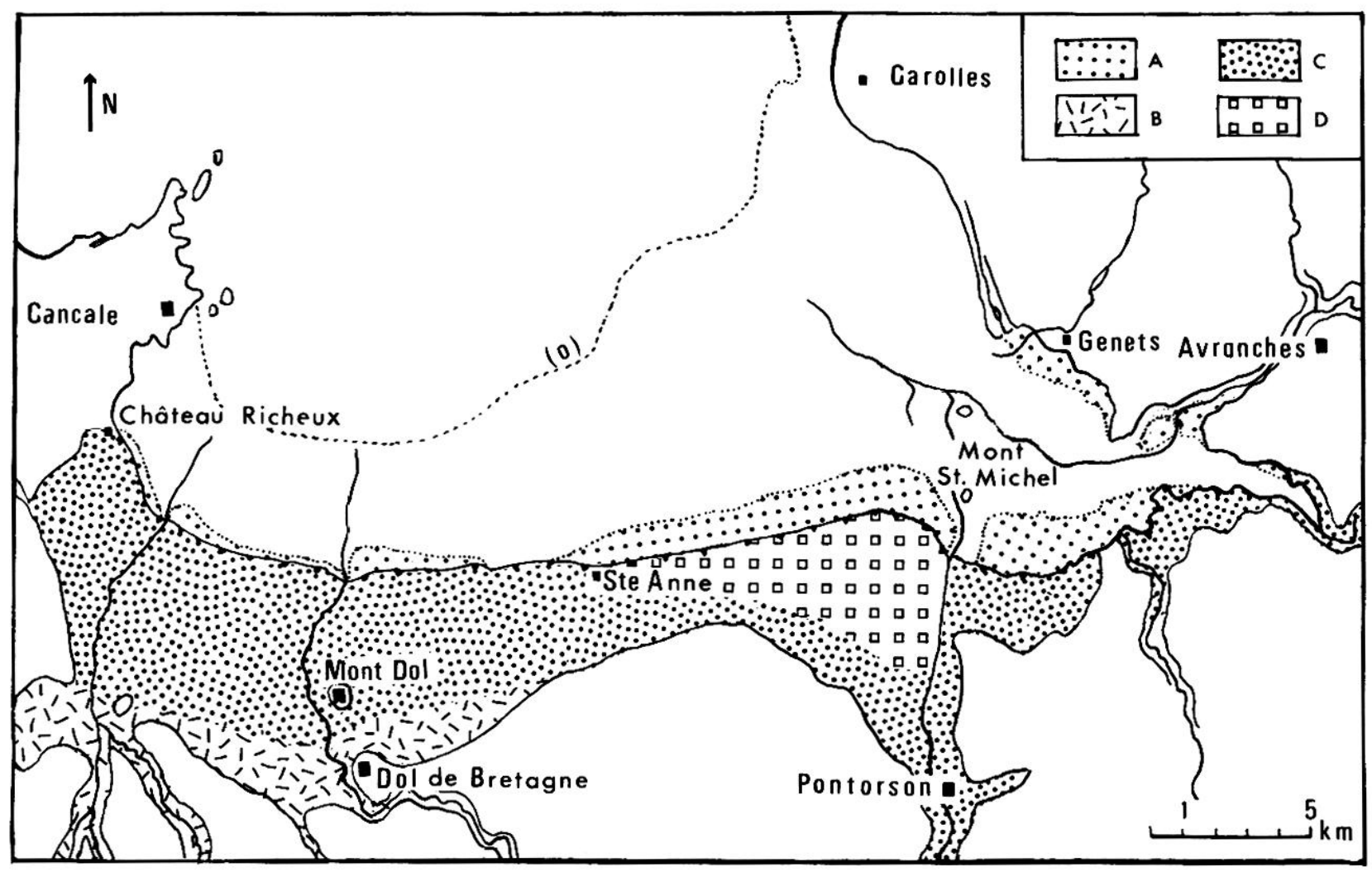

Fig. 1. - Cadre géographique du marais maritime de Dol-de-Bretagne (A. schorre; B. marais noir, tourbeux; C. marais blanc; D. polder. 


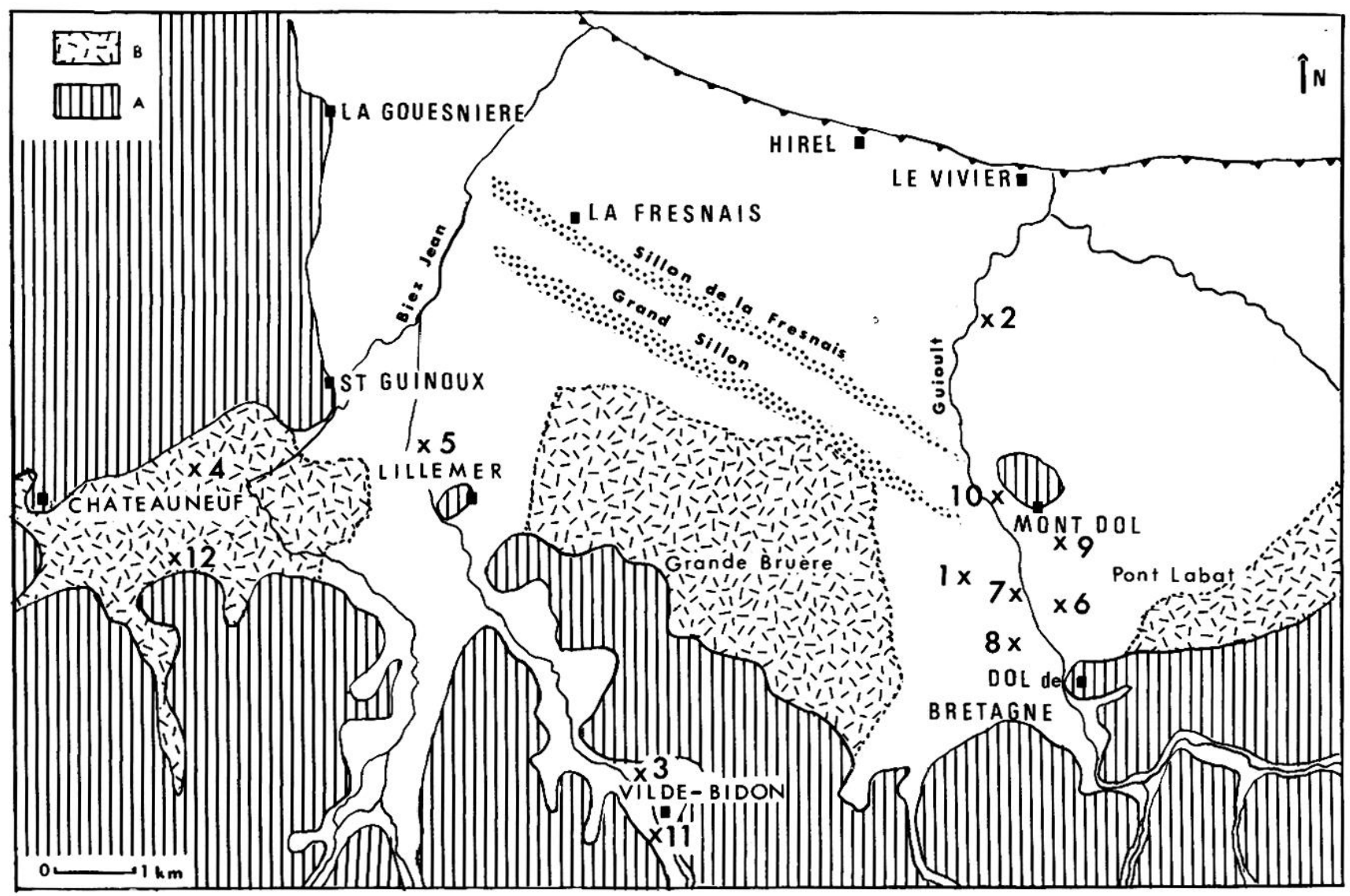

Fig. 2. - Localisation des sondages dans la partie occidentale du Marais de Dol-de-Bretagne (A. plateau limitant au Sud le marais maritime; B. marais tourbeux).

Ces sondages ont été implantés essentiellement dans le marais blanc, le long du Guioult (S. 2, 10, 7, 8) ainsi que de part et d'autre de ce ruisseau (S. 9, 6 et 1). Quelques sondages ont également été réalisés dans le fond du marais, dans la dépression tourbeuse de Châteauneuf et le long du Biez Jean, près de VildéBidon, afin d'obtenir les stades les plus élevés de l'invasion marine.

\section{Lithologie du remblaiement du marais (fig. 3 et 4 )}

Le socle a été atteint dans tous les sondages, sauf aux points d'implantation 2 et 5 où celui-ci se trouvait à plus de 20 mètres de profondeur.

Sous le marais, le substratum est constitué de schistes briovériens très altérés. Il se présente comme une zone plate, descendant en pente douce vers le Nord, sillonnée par les thalwegs prolongeant ceux qui entaillent au Sud l'abrupt de l'ancienne falaise. Le tracé de ces thalwegs, autant que l'on puisse en juger par les résultats des douze sondages, correspond à ceux des cours actuels du Guioult et du Biez Jean.

\section{1) Le Weichselien - les sédiments préflandriens}

Les dépôts périglaciaires weichseliens sont formés de coulées limoneuses emballant parfois des blocs et des cailloutis. Ils se sont maintenus au pied de la falaise fossile et sur le plateau.

Dans les vallées ces limons sont surmontés de sables et de galets fluviatiles qui se sont mis en place, semble-t-il, assez tardivement au cours du Weichselien. Dans la vallée du Biez Jean la sédimentation lacustre se poursuit par le dépôt d'une boue organique renfermant une flore tardiglaciaire à plantes herbacées dominantes, Pinus et Betula clairsemés et datée du Dryas récent : S. 11, Gif $954=11600 \pm 650$ ans et Gif $1834=$ $10950 \pm 230$ ans (échantillon prélevé à $7,95 \mathrm{~m}$ de profondeur). La matière organique, provenant de l'échantillon prélevé à la base du sondage 8 près du Mont Dol, datée de $10100 \pm 230$ ans (Gif 1833), s'est également déposée au Tardiglaciaire.

Un limon de colluvionnement recouvre les sables fluviatiles et la boue organique (S. 11, 3). Il représente la sédimentation de la fin du Tardiglaciaire et du début du Flandrien.

\section{2) Le Flandrien - la sédimentation littorale}

Dans le marais maritime, près du Mont Dol, la sédimentation flandrienne débute, dans les thalwegs, par une argile grise très riche en matière organique. $\mathrm{Ce}$ dépôt légèrement saumâtre s'est formé en avant de la transgression flandrienne; il est l'équivalent de la tourbe inférieure des Pays-Bas (Lower Peat). Au-dessus la 
LE LONG DU GUIOULT

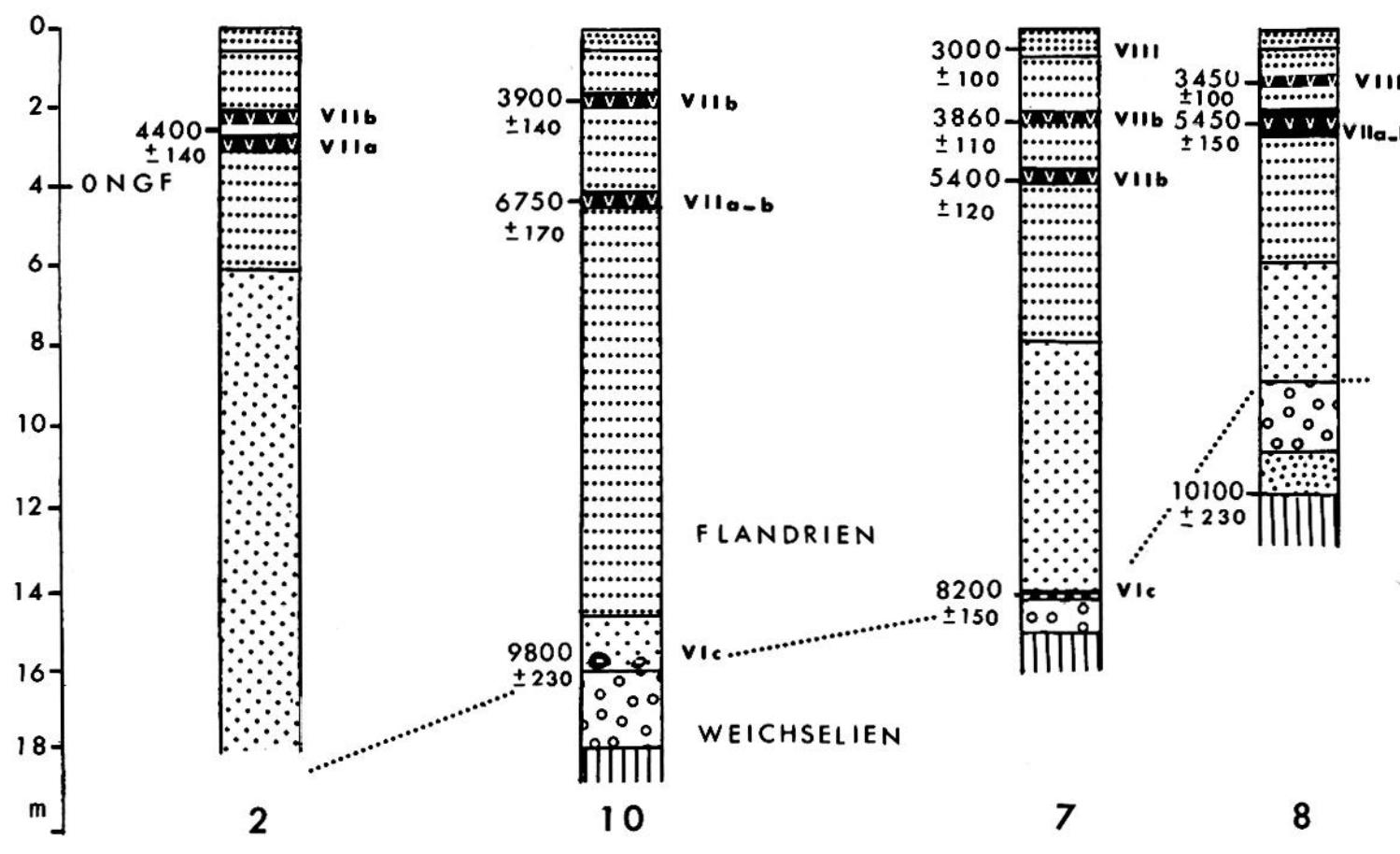

A LEST DU GUIOULT

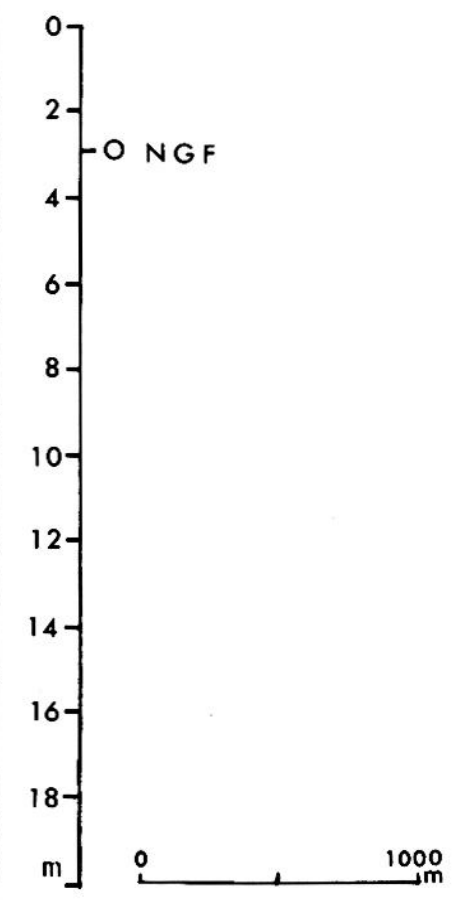

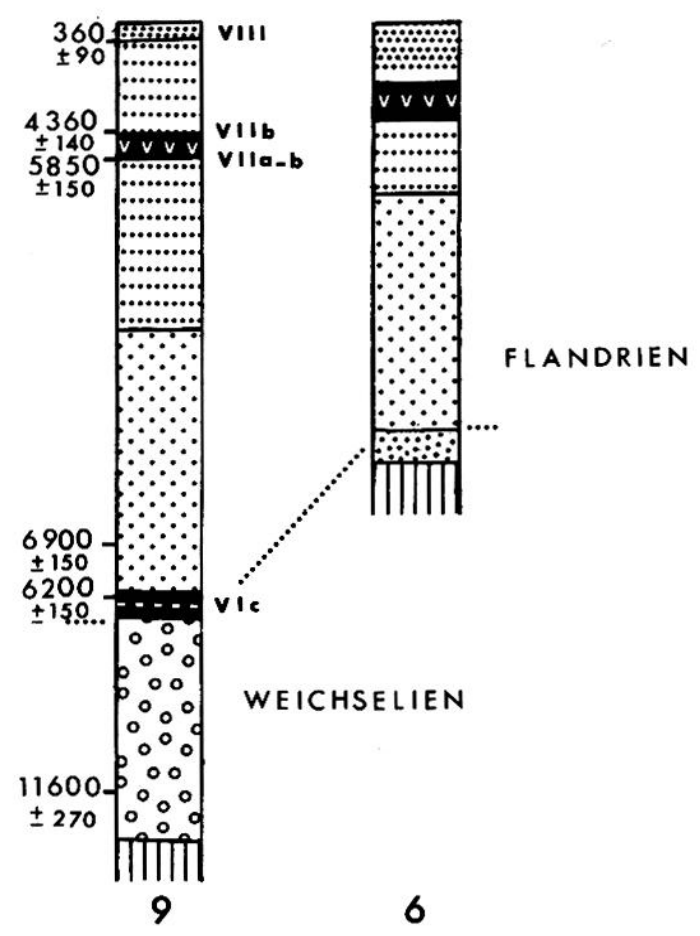

A L'OUEST DJ GUIOULT

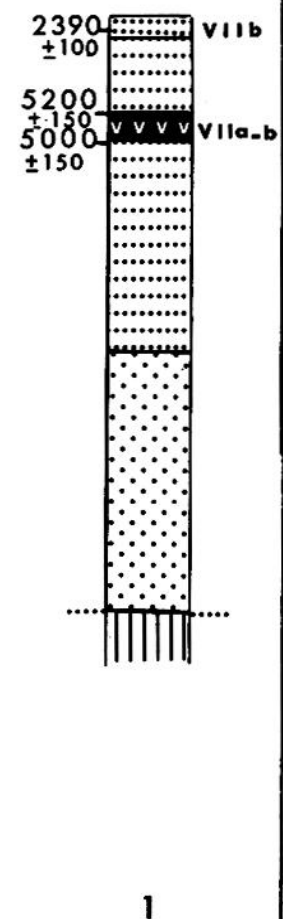

Fig. 3. - Sondages dans le Marais blanc : lithologie, datation radiocarbone et datation pollinique. 


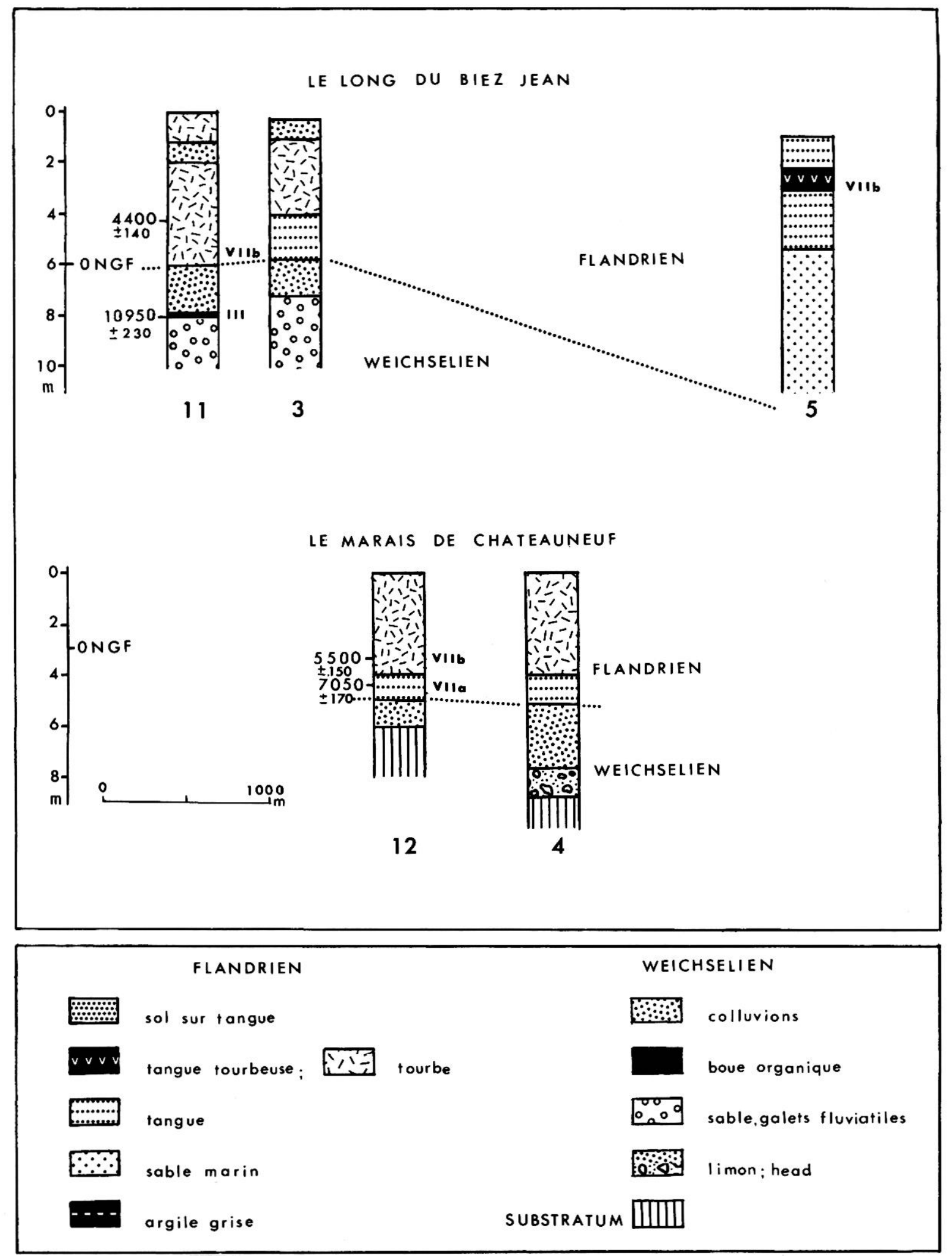

Fig. 4. - Sondages dans le Marais noir : lithologie, datation radiocarbone, datation pollinique.

sédimentation marine est représentée par des sédiments intertidaux : sables et tangues. En arrière du cordon littoral actuel l'épaisseur de l'ensemble est en moyenne de $14 \mathrm{~m}$. Au sommet, au-dessus de $4 \mathrm{~m}$ de profondeur, s'intercalent deux couches tourbeuses aux abords du
Guioult, une seule de part et d'autre de ce ruisseau (fig. 3). Le sol actuel est établi sur de la tangue.

Dans le marais de Châteauneuf les sédiments intertidaux ont en moyenne $1 \mathrm{~m}$ d'épaisseur (fig. 4). Dans 
la vallée du Biez Jean, ils atteignent Vildé-Bidon en amont (S. 3) mais ne dépasse guère ce point (S. 11).

a. Les sables.

Le dépôt marin inférieur est un sable grossier, bien classé et coquillier (Cardium edule en abondance). Sa mise en place est accompagnée d'érosion ; la base de ce dépôt renferme des galets d'argile grise arrachés au niveau tourbeux inférieur (S. 10).

b. La tangue.

La tangue est un sable très fin plus ou moins argileux, calcaire et qui présente une structure litée caractéristique. C'est un dépôt spécial, particulièrement bien représenté dans la baie du Mont-Saint-Michel ; il est lié aux mouvements de la marée.

\section{c. Les niveaux tourbeux.}

Les niveaux tourbeux qui s'intercalent dans la sédimentation marine littorale sont des tourbes très riches en éléments clastiques dérivés des sédiments marins. Ce sont des tourbes formées dans des marais littoraux ouverts, à Chénopodiacées, évoluant localement et temporairement en marais à Cypéracées. Le stade de la Phragmitaie n'est jamais atteint.

La partie sud-ouest du marais est isolée très tôt de l'influence marine. Dans la dépression de Châteauneuf, au marais à Cypéracées dominantes, succède une tourbière à Sphaignes et Ericacées.

Résultats palynologiques et datation par le $14 \mathrm{C}$ (fig. 3 et 4 ; tabl. I)

L'étude palynologique complète de tous les sédiments tourbeux a déjà été publiée (M.T.MorzadecKerfourn, 1974). Seules seront exposées ici les conclusions stratigraphique et écologique de cette étude.

Le tableau I permet de confronter pour l'ensemble du marais les dates $14 \mathrm{C}$ et les datations proposées par la palynologie. Le tableau II présente les âges $14 \mathrm{C}$ attribués aux limites des zones polliniques en Armorique (M.T. Morzadec-Kerfourn, 1974).

\section{1) Le Marais de Châteauneuf (fig. 4)}

- Datation pollinique.

Les sédiments intertidaux présents entre 5 et $4 \mathrm{~m}$ de profondeur, renferment une végétation caractéristique de l'Atlantique (zone VIla de $\mathrm{H}$. Godwin) : faible représentation d'Alnus, présence constante d'Ulmus. Ils témoignent d'une extension importante de la mer vers le Sud à cette époque dans le fond de la baie.

La tourbe qui surmonte ces sables présente encore des caractères saumâtres jusqu'à $3,45 \mathrm{~m}$ de profondeur. Ce dépôt date de la période de transition AtlantiqueSubboréal : égale représentation de Quercus et d'Alnus, légère régression d'Ulmus.

Au-dessus les sédiments tourbeux lacustres se sont formés du Subboréal à l'Actuel.

- Datation radiocarbone.

sondage 12, Gif $\begin{aligned} 1838= & 7050 \pm 170 \text { années à } 4,50 \mathrm{~m}, \\ & \text { ancien schorre. } \\ \text { Gif } 955= & 5600 \pm 150 \text { années à } 3,90 \mathrm{~m}, \\ & \begin{array}{l}\text { début de la sédimentation tour- } \\ \text { beuse. }\end{array}\end{aligned}$

Gif $1837=5500 \pm 150$ années à $3,45 \mathrm{~m}$, fin de l'influence marine dans la dépression de Châteauneuf.

\section{2) Le Biez Jean (fig. 4)}

En amont, au-dessus des colluvions tardiglaciaires, l'influence marine se traduit par le dépôt de tangue (entre $6 \mathrm{~m}$ et $4 \mathrm{~m}$ de profondeur dans le sondage 3 ) ou par le développement d'une végétation halophile dans la tourbe (entre $6,10 \mathrm{~m}$ et $4,20 \mathrm{~m}$ de profondeur dans le sondage 11). Au niveau du sondage 5 la sédimentation est marine jusqu'à $2,50 \mathrm{~m}$ de profondeur.

- Datation pollinique.

L'ensemble des tourbes qui colmatent là vallée du Biez Jean date du Subboréal: abondance d'Alnus, présence de Fagus.

- Datation radiocarbone.

sondage 11, Gif $953=4800 \pm 140$ années, bois à $6,10 \mathrm{~m}$, début de la formation tourbeuse.

Gif $1835=4400 \pm 140$ années, à $4,20 \mathrm{~m}$, fin de l'influence marine.

Gif $958=3100 \pm 110$ années, à $2 \mathrm{~m}$, sommet de la première formation tourbeuse, sous le colluvion récent.

Gif $957=3100 \pm 110$ années, à $1,20 \mathrm{~m}$, tase de la seconde formation tourbeuse.

\section{3) Le Marais Blanc, près du Mont Dol (fig. 3)}

Dans le Marais Blanc le début de la sédimentation flandrienne date du Boréal. On retrouve dans tous les sondages la même succession stratigraphique.

a. L'argile noire de base.

L'argile noire apparaît près du Mont Dol, au niveau du Guioult (S. 7 et 9). Plus au Nord (S. 10) elle est présente, à la base des sables marins, sous forme de galets.

- Datation pollinique : Boréal terminal, VIc.

L'abondance de Corylus, le taux élevé de Pinus, la faible représentation d'Alnus confèrent un âge Boréal terminal à l'ensemble de cette formation (zone VIc de H. Godwin).

- Datation radiocarbone.

sondage 7 , Gif $2188=8200 \pm 150$ années, à $14 \mathrm{~m}$ ( $-10 \mathrm{~m}$ N.G.F.). 


\begin{tabular}{|c|c|c|c|c|c|c|c|}
\hline \multirow{2}{*}{$\begin{array}{c}\text { Numéro } \\
\text { du sondage }\end{array}$} & \multicolumn{2}{|c|}{ Altitude du dépôt en m } & \multicolumn{2}{|c|}{ Date $14 \mathrm{C}$} & \multirow{2}{*}{$\begin{array}{l}\text { Nature du } \\
\text { dépôt }\end{array}$} & \multirow{2}{*}{$\begin{array}{l}\text { Zone } \\
\text { pollinique }\end{array}$} & \multirow{2}{*}{ Caractères de la végétation } \\
\hline & $\begin{array}{c}\text { par rapport } \\
\text { à la } \\
\text { surface }\end{array}$ & $\begin{array}{c}\text { par rapport } \\
\text { au N.G.F. }\end{array}$ & $\begin{array}{c}\text { Numéro } \\
\text { Gif }\end{array}$ & Age & & & \\
\hline $\begin{array}{l}\text { S } 11 \\
\quad \grave{a} \\
+6 \text { m N.G.F. }\end{array}$ & $\begin{array}{l}7,95 \\
6,10 \\
4,20 \\
2,00 \\
1,20\end{array}$ & $\begin{array}{l}-1,95 \\
-\quad 0,10 \\
+\quad 1,80 \\
+\quad 4,00 \\
+\quad 4,80\end{array}$ & $\begin{array}{r}1834 \\
953 \\
1853 \\
958 \\
957\end{array}$ & $\begin{array}{r}10950 \pm 230 \\
4800 \pm 140 \\
4400 \pm 140 \\
3100 \pm 110 \\
3100 \pm 110\end{array}$ & $\begin{array}{c}\text { eau douce } \\
\pm \text { saumâtre } \\
\text { fin saumâtre } \\
\text { eau douce } \\
\text { eau douce }\end{array}$ & $\begin{array}{c}\text { III } \\
\text { VIIb } \\
\text { VIIb } \\
\text { VIIb-VIII } \\
\text { VIIb-VIII }\end{array}$ & $\begin{array}{l}\text { Pinus dominant, Betula, Corylus, Juniperus. } \\
\text { Alnus dominant, Quercus, Tilia, Ulmus. } \\
\text { Alnus dominant, Quercus, Tilia. } \\
\text { Alnus dominant, Quercus, Fagus. } \\
\text { Alnus dominant, Quercus, Tilia, Fagus. }\end{array}$ \\
\hline $\begin{array}{c}\mathrm{S} 12 \\
\mathrm{a} \\
+2,2 \text { m N.G.F. }\end{array}$ & $\begin{array}{l}4,50 \\
3,90 \\
3,50\end{array}$ & $\begin{array}{l}-2,30 \\
-\quad 1,70 \\
-\quad 1,30\end{array}$ & $\begin{array}{c}1838 \\
955 \\
1837\end{array}$ & $\begin{array}{l}7050 \pm 170 \\
5600 \pm 150 \\
5500 \pm 150\end{array}$ & $\begin{array}{c}\text { saumâtre } \\
\text { saumâtre } \\
\text { fin saumâtre }\end{array}$ & $\begin{array}{l}\text { VIIa } \\
\text { VIIa-VIIb } \\
\text { VIIb }\end{array}$ & $\begin{array}{l}\text { Quercus dominant, Alnus, Ulmus, Tilia. } \\
\text { Quercus = Alnus, Ulmus, Tilia. } \\
\text { Quercus = Alnus, Ulmus, Tilia (Fagus). }\end{array}$ \\
\hline $\begin{array}{c}\text { S } 7 \\
\quad \mathrm{a} \\
+4 \mathrm{~m} \mathrm{N.G.F.}\end{array}$ & $\begin{array}{r}14,00 \\
3,50 \\
2,00 \\
0,80\end{array}$ & $\begin{array}{r}-10,00 \\
-\quad 0,50 \\
+\quad 2,00 \\
+\quad 3,20\end{array}$ & $\begin{array}{l}2188 \\
2187 \\
2186 \\
2185\end{array}$ & $\begin{array}{l}8200 \pm 150 \\
5400 \pm 120 \\
3860 \pm 110 \\
3000 \pm 100\end{array}$ & $\begin{array}{l}\text { eau douce } \\
\text { eau douce } \\
\pm \text { saumâtre } \\
\pm \text { saumâtre }\end{array}$ & $\begin{array}{l}\text { VIc } \\
\text { VIIb } \\
\text { VIIb } \\
\text { fin VIIb }\end{array}$ & $\begin{array}{l}\text { Corylus, Quercus dominants, Pinus. } \\
\text { Alnus dominant, Quercus, Ulmus, Tilia (Fagus). } \\
\text { Alnus dominant, Quercus, Tilia. } \\
\text { Alnus dominant, Pinus, Quercus, Tilia. }\end{array}$ \\
\hline $\begin{array}{l}\text { S } 10 \\
\quad \text { à } \\
+4 \mathrm{~m} \mathrm{N.G.F}\end{array}$ & $\begin{array}{r}16,00 \\
4,20 \\
1,85\end{array}$ & $\begin{array}{l}-12,00 \\
-\quad 0,20 \\
+\quad 2,15\end{array}$ & $\begin{array}{l}952 \\
951 \\
950\end{array}$ & $\begin{array}{l}9800 \pm 230 \\
6750 \pm 170 \\
3900 \pm 140\end{array}$ & $\begin{array}{l}\text { saumâtre } \\
\text { saumâtre } \\
\text { saumâtre }\end{array}$ & $\begin{array}{l}\text { VIc } \\
\text { fin VIIa } \\
\text { VIIb }\end{array}$ & $\begin{array}{l}\text { Corylus, Quercus dominants, Pinus. } \\
\text { Quercus dominant, Alnus, Ulmus, Tilia. } \\
\text { Alnus dominant, Quercus, Tilia (Fagus). }\end{array}$ \\
\hline $\begin{array}{c}\text { S } 8 \\
\quad \text { à } \\
+3 \text { m N.G.F. }\end{array}$ & $\begin{array}{r}12,00 \\
2,75 \\
2,00 \\
1,20\end{array}$ & $\begin{array}{l}-\quad 9,00 \\
+\quad 0,25 \\
+\quad 1,00 \\
+\quad 1,80\end{array}$ & $\begin{array}{c}1833 \\
948 \\
947 \\
2189\end{array}$ & $\begin{array}{rr}10 & 100 \pm 230 \\
5 & 450 \pm 150 \\
5 & 450 \pm 150 \\
3 & 450 \pm 100\end{array}$ & $\begin{array}{c}\text { argile } \\
\text { altération } \\
\text { saumâtre } \\
\text { saumâtre } \\
\pm \text { saumâtre }\end{array}$ & $\begin{array}{l}\text { début VIIb } \\
\text { VIIb } \\
\text { vIIb }\end{array}$ & $\begin{array}{l}\text { Quercus dominant, Alnus, Tilia. } \\
\text { Alnus dominant, Quercus, Tilia. } \\
\text { Alnus dominant, Quercus, Tilia. }\end{array}$ \\
\hline $\begin{array}{c}S \mathrm{I} \\
\mathrm{a} \\
+2,3 \mathrm{~m} \mathrm{~N} \text {.G.F. }\end{array}$ & $\begin{array}{l}6,30 \\
2,55 \\
2,25 \\
0,50\end{array}$ & $\begin{array}{r}-\quad 2,00 \\
-\quad 0,25 \\
+\quad 0,05 \\
+\quad 11,80\end{array}$ & $\begin{array}{r}946 \\
944 \\
943 \\
2184\end{array}$ & $\begin{array}{l}6800 \pm 170 \\
5000 \pm 150 \\
5200 \pm 150 \\
2390 \pm 100\end{array}$ & $\begin{array}{c}\text { sable marin } \\
\text { saumâtre } \\
\text { saumâtre } \\
\pm \text { saumâtre }\end{array}$ & $\begin{array}{l}\text { VIIa } \\
\text { VIIa } \\
\text { fin VIIb }\end{array}$ & $\begin{array}{l}\text { Quercus dominant, Alnus, Ulmus, Tilia. } \\
\text { Quercus dominant, Alnus, Ulmus, Tilia (Fagus). } \\
\text { Alnus dominant, Quercus, Pinus. }\end{array}$ \\
\hline $\begin{array}{c}\text { S } 9 \\
\quad \grave{a} \\
+3 \text { m N.G.F. }\end{array}$ & $\begin{array}{r}18,00 \\
13,20 \\
12,30 \\
3,20 \\
3,10 \\
2,80 \\
2,60 \\
0,30\end{array}$ & $\begin{array}{l}-15,00 \\
-10,20 \\
-\quad 9,30 \\
-\quad 0,20 \\
-\quad 0,10 \\
+\quad 0,20 \\
+\quad 0,40 \\
+\quad 2,70\end{array}$ & $\begin{array}{r}942 \\
941 \\
940 \\
937 \\
1834 \\
936 \\
935 \\
931\end{array}$ & $\begin{array}{r}11600 \pm 270 \\
6200 \pm 150 \\
6900 \pm 150 \\
4250 \pm 140 \\
5850 \pm 150 \\
4480 \pm 140 \\
4360 \pm 140 \\
360 \pm 90\end{array}$ & $\begin{array}{c}\text { sable } \\
\text { fluviatile } \\
\pm \text { saumâtre } \\
\text { sable marin } \\
\text { saumâtre } \\
\text { saumâtre } \\
\text { saumâtre } \\
\text { saumâtre } \\
\text { sol }\end{array}$ & $\begin{array}{c}\text { VIc } \\
\text { VIIa } \\
\text { VIIa } \\
\text { VIIa } \\
\text { VIIa-VIIb } \\
\text { VIII }\end{array}$ & $\begin{array}{l}\text { Corylus, Betula dominants, Pinus, Quercus. } \\
\text { Quercus dominant, Alnus, Tilia, Ulmus. } \\
\text { Quercus dominant, Alnus, Tilia, Ulmus. } \\
\text { Quercus dominant, Alnus, Tilia, Ulmus. } \\
\text { Quercus = Alnus, Tilia, Ulmus (Fagus). } \\
\text { Alnus dominant, Pinus, Quercus. }\end{array}$ \\
\hline $\begin{array}{c}\text { S } 2 \\
\quad \grave{a} \\
+6 \text { m N.G.F. }\end{array}$ & 2,50 & $+3,50$ & 1879 & $4400 \pm 140$ & \pm saumâtre & VIIa & Quercus dominant, Alnus, Tilia, Ulmus. \\
\hline
\end{tabular}

Tableau I. - Résumé des données polliniques et $14 \mathrm{C}$ des sédiments du marais de Dol-de-Bretagne.

sondage 9 , Gif $941=6200 \pm 150$ années, à 13,20 m ( $-10,20 \mathrm{~m}$ N.G.F.).

sondage 10, Gif $952=9800 \pm 230$ années, à $16 \mathrm{~m}$ ( $-12 \mathrm{~m}$ N.G.F.), galets d'argile.

b. Le premier niveau tourbeux.

En arrière du cordon actuel une première lentille tourbeuse est interstratifiée dans la tangue à une altitude proche du niveau moyen des marées, entre $-0,50$ et $+0,40 \mathrm{~m}$ N.G.F. Son épaisseur est variable : $20 \mathrm{~cm}$ près du Guioult, 60 à $70 \mathrm{~cm}$ à l'Ouest de ce ruisseau.

- Datation pollinique: Fin Atlantique - début Subboréal, VIIa-VIIb.

La végétation conservée dans ce niveau présente les variations caractéristiques du passage de l'Atlantique (zone VIIa) au Subboréal (zone VIIb) ; Quercus décroît au profit d'Alnus, la représentation de Tilia et surtout d'Ulmus devient irrégulière, Fagus apparaît. Le passage entre ces deux zones s'observe dans la tourbe à l'Ouest du Guioult (S. 1 et 8) et à l'Est (S. 9). Près du Guioult la tourbe date tantôt de l'Atlantique (S. 10,2) tantôt du Subboréal (S. 7).

Près du Biez Jean (S. 5) l'unique lentille tourbeuse est également subboréale comme la tourbe saumâtre du marais de Châteauneuf.

L'évolution de cette formation tourbeuse s'effectue à partir d'un marais à Chénopodiacées vers un marais à Cypéracées et Graminées.

- Datation radiocarbone.

sondage 1 , Gif $943=5200 \pm 150$ années, à $2,25 \mathrm{~m}$ $(+0,05 \mathrm{~m}$ N.G.F.), zone VIIb, 
Gif $944=5000 \pm 150$ années, à $2,55 \mathrm{~m}$ $(-0,25 \mathrm{~m}$ N.G.F.), zone VIla. sondage 7, Gif $2187=5400 \pm 120$ années, à $3,50 \mathrm{~m}$ $(-0,50 \mathrm{~m}$ N.G.F.), zone VIIb. sondage 8, Gif $947=5450 \pm 150$ années, à $2,00 \mathrm{~m}$ $(+1,00 \mathrm{~m}$ N.G.F.), zone VIIb.

Gif $948=5450 \pm 150$ années, à $2,75 \mathrm{~m}$ $(+0,25 \mathrm{~m}$ N.G.F.), zone VIlb. sondage 9 , Gif $935=4360 \pm 140$ années, à $2,60 \mathrm{~m}$ $(+0,40 \mathrm{~m}$ N.G.F. $)$, zone VIIb.

Gif $936=4480 \pm 140$ années, à $2,80 \mathrm{~m}$ $(+0,20 \mathrm{~m}$ N.G.F. $)$, zone VIIa.

Gif $937=4250 \pm 140$ années, à $3,20 \mathrm{~m}$ $(-0,10 \mathrm{~m}$ N.G.F. $)$, zone VIIa.

Gif $1834=5850 \pm 150$ années, à $3,10 \mathrm{~m}$ $(-0,20 \mathrm{~m}$ N.G.F.), zone VIIa.

sondage 10 , Gif $951=6750 \pm 150$ années, à $4,20 \mathrm{~m}$ $(-0,20 \mathrm{~m} \quad$ N.G.F. $)$, fin zone VIIa.

c. La seconde lentille tourbeuse.

la seconde lentille tourbeuse qui apparaît entre $+2,15 \mathrm{~m}$ et $+1,80 \mathrm{~m}$ N.G.F., est moins importante que la précédente. Elle est surtout visible au niveau du chenal du Guioult (S. 2, 7, 8, 10).

- Datation pollinique : Subboréal.

L'abondance d'Alnus, la présence constante de Tilia sont caractéristiques du Subboréal (zone VIIb).
- Datation radiocarbone.

sondage 7, Gif $2186=3860 \pm 110$ années, à $2,00 \mathrm{~m}$ $(+2,00 \mathrm{~m}$ N.G.F.).

sondage 8 , Gif $2189=3450 \pm 100$ années, à $1,20 \mathrm{~m}$ $(+1,80$ m N.G.F.).

sondage 10 , Gif $950=3,900 \pm 140$ années, à $1,85 \mathrm{~m}$ $(+2,15 \mathrm{~m}$ N.G.F.).

d. La tangue supérieure.

- Datation pollinique (Subboréal - Subatlantique).

Un sol s'est établi près de la surface, au-dessus de la tangue. Sa formation correspond à plusieurs horizons stratigraphiques qui s'échelonnent de la fin du Subboréal au Subatlantique, en liaison avec la construction des cordons littoraux: Le Grand Sillon à la fin du Subboréal et le Sillon de la Fresnais au Subatlantique.

En arrière du Grand Sillon, en bordure du marais de la Grande Bruère ( $S .1$ et 8 ) le début de la tangue supérieure date de la fin du Subboréal. Ailleurs ce sol est moins caractéristique et sa formation se situerait à la fin du Subboréal ou au tout début du Subatlantique.

- Datation radiocarbone.

sondage 7, Gif $2185=3000 \pm 100$ années, à $0,80 \mathrm{~m}$ (+3,2 m N.G.F.).

sondage 9 , Gif $931=360 \pm 90$ années, à $0,30 \mathrm{~m}$ $(+2,70 \mathrm{~m}$ N.G.F.).

sondage 1, Gif $2184=2390 \pm 100$ années, à $0,50 \mathrm{~m}$ $(+1,80$ m N.G.F.).

\begin{tabular}{|c|c|}
\hline $\begin{array}{l}\text { Périodes climatiques } \\
\text { (Blytt et Sernander) }\end{array}$ & Zones polliniques \\
\hline Subat lantique & $\begin{array}{l}\text { Quercus, Alnus, Fagus } \\
\text { vIII }\end{array}$ \\
\hline Subboréal-Subatlantique & $\begin{array}{l}\text { Quercus, Alnus (Tilia, Fagus) } \\
\text { vIIb-VIII }\end{array}$ \\
\hline Subboréal & $\begin{array}{c}\text { Alnus, Quercus, Tilia } \\
\text { vIIb }\end{array}$ \\
\hline Atlantique & $\begin{array}{l}\text { Quercus, Alnus, Tilia, Ulmus } \\
\text { vIIa }\end{array}$ \\
\hline \multirow{2}{*}{ Boréa1 } & $\begin{array}{c}\text { Corylus, Quercus, Pinus (UImus) } \\
\text { VI }\end{array}$ \\
\hline & $\begin{array}{c}\text { Pinus, Betula, Quercus } \\
\mathrm{v}\end{array}$ \\
\hline Préboréal & $\begin{array}{l}\text { Betula, Pinus } \\
\text { IV }\end{array}$ \\
\hline
\end{tabular}

Tableau II. - Evolution de la végetation armoricaine du Flandrien; limites radiochronologiques des zones polliniques. 
En résumé, si dans le marais noir les analyses radiométriques et palynologiques ont donné des résultats concordants, dans le marais blanc ceux-ci sont moins satisfaisants (tableau I). Une date radiocarbone sur trois est "trop vieille" ou "trop récente" si l'on s'en tient aux définitions de l'analyse pollinique (tableau II). Pour deux sondages l'accord est bon entre l'âge $14 \mathrm{C}$ et la zone pollinique et ceci pour tout le profil (S. 7 et S. 8).

Pour le sondage 10 tout est trop vieux tandis que pour les sondages 1 et 9 tout est trop récent excepté les résultats obtenus sur débris de coquilles (Gif 940).

\section{COMMENTAIRES CONCERNANT CES RESULTATS}

Ces divergences nous ont conduites à examiner en détail quelles sont les causes qui peuvent soit fausser certains âges $14 \mathrm{C}$, soit entâcher d'erreur certaines analyses polliniques.

\section{1) La datation radiocarbone}

La diversité des terrains traversés nous a amenées à utiliser pour les datations des matériaux forts divers: tourbe et tangue tourbeuse, tangue, sable coquillier.

a. La tourbe.

La datation des tourbes est un problème classique et le traitement chimique préliminaire qu'elles doivent subir est aussi théoriquement bien connu : il consiste à éliminer les acides humiques qui les imprègnent pour ne travailler que sur le résidu de matière végétale. Les acides humiques en effet, s'ils proviennent essentiellement de la décomposition in situ des végétaux qui constituent la tourbe, peuvent aussi venir de niveaux supérieurs, donc plus jeunes que celui qu'on a l'intention de dater; on aurait alors un rajeunissement de l'échantillon. Mais dans le cas des tourbes très riches en matières humiques, il est difficile de prétendre s'en séparer rigoureusement et en particulier dans le cas d'échantillons peu abondants ou dont la décomposition est très avancée, il est pratiquement impossible de poursuivre ce traitement jusqu'à leur élimination complète car on risque alors de perdre la totalité du prélèvement. Il peut aussi y avoir apport de carbone récent par suite de pénétration profonde des végétaux de surface. Il n'est donc pas impossible de trouver quelquefois un léger rajeunissement d'un niveau tourbeux.

On voit moins de possibilités de vieillissement que de rajeunissement pour une tourbe si ce n'est par apport de carbone organique ancien au cours de sa formation. Cet effet serait alors d'autant plus sensible qu'on aurait affaire non plus à une vraie tourbe mais à un sédiment plus ou moins tourbeux, donc plus ou moins riche en carbone et par suite plus facilement contaminable (I.U. Olsson, 1972).

Tout risque de pollution par du calcaire ancien est éliminé à la suite du traitement acide préliminaire subi par l'échantillon. L'absence de Carbone d'origine minérale peut être démontrée par la mesure du rapport isotopique $\frac{\mathrm{C} 13}{\mathrm{C} 14}$ du carbone. Le problème s'est posé, dans le marais de Dol, pour la base du sondage 8 où aucun pollen n'est venu confirmé la date $10100 \pm 230$ années (Gif 1833) obtenue ; ce sédiment argileux est très pauvre en carbone organique : $0,10 \%$, et l'on pouvait craindre que le carbone daté ne soit pas en totalité d'origine organique mais qu'il soit en partie le résidu de carbone minéral mal éliminé lors de la préparation chimique. La mesure du $\delta \mathrm{C} 13(-27,2 \% 0)$ a prouvé de façon irréfutable qu'il s'agıssait bien de carbonate provenant de matière organique ; un carbone minéral aurait donné un $\delta \mathrm{C} 13$ voisin de $0 \%$ c'est-àdire proche du $\delta \mathrm{C} 13$ de carbonate marin de référence (1)

\section{b. Le sable.}

Les coquilles, quelques fois présentes dans les sables, étaient toujours trop petites et trop peu abondantes pour être isolées et datées. C'est donc la partie calcaire dans sa totalité qui a été utilisée ; partie qui comprend essentiellement des débris de coquilles mais aussi, peutêtre, une partie détritique constituée par du calcaire ancien. Par ailleurs, remarquons que même en datant les coquilles on ne date pas précisément dans tous les cas la mise en place du sédiment. Ces raisons font qu'il faut s'attendre à un vieillissement pour ce genre de matériau.

\section{c. La tangue.}

L'abondance des tangues dans cette zone nous a conduit à tenter leur datation. L'utilisation de la partie calcaire était à priori, pour les raisons invoquées pour les sables coquilliers, bien aléatoire. L'expérience a montré qu'elle était absolument inutilisable, le calcaire détritique étant en proportion importante dans ces formations littorales (J. Bourcart et G. Boillot, 1960). Dans le sondage 9 , entre 0,60 et $2,50 \mathrm{~m}$ de profondeur, la tangue du marais a un âge apparent de 8500,7300 et 10900 ans alors que son âge réel se situerait logiquement entre 3600 et 3000 ans.

\section{2) L'analyse pollinique}

Les grains de pollens et les spores ont une paroi très résistante qui permet leur conservation dans les sédi-

(1) C 13 représente la différence entre la composition isotopique d'un carbone quelconque et la composition isotopique du carbone standard qui est une calcite provenant d'un rostre de Belemnite du Crétacé supérieur ; $\delta \mathrm{C} 13$ varie couramment entre des valeurs proches de zéro pour les calcaires marins et des valeurs voisines de $-25 \%$ pour les végétaux. 
ments maintenus à l'abri de l'oxydation. Non seulement les tourbes mais aussi les vases, les argiles, les sables fins peuvent en contenir. La fréquence des pollens dans ces milieux divers est telle que l'analyse pollinique est devenu un bon instrument de la stratigraphie du Quaternaire. Le fondement en est simple. Après le retrait des glaciers du Nord de l'Europe et le recul de la zone périglaciaire, la végétation forestière s'installe progressivement pour arriver à son état actuel. L'abondance des dépôts tourbeux flandriens, en toutes régions, permet de suivre ce cheminement, d'établir une zonation pollinique et de préciser ainsi la chronologie relative des différentes formations.

Cette méthode, pour être efficace, suppose que les pollens piégés dans le sédiment sont contemporains de la mise en place de celui-ci. Cependant trois cas principaux de pollution par du pollen étranger peuvent être envisagés : la pluie pollinique actuelle, la migration de pollens de surface à travers le sédiment et enfin le remaniement de matériel plus ancien.

a - L'apport accidentel de pollens frais est aisément décelé, la technique de préparation, ne faisant pas intervenir l'acétolyse, n'agit pas sur le contenu pollinique.

b - La pénétration de grains de pollens dans un sédiment varie selon la nature de celui-ci. Grâce à l'eau de circulation et sous l'influence de facteurs biologiques, les pollens pénètrent aisément dans les sols sableux (G.W. Dimbleby, 1962 ; A.V. Munaut, 1967). La vitesse varie de 1 à $5 \mathrm{~cm}$ par siècle; mais l'infiltration est stoppée dans les sols ensevelis. Selon B. Bastin (1971) dans les dépôts plus fins, limoneux et loessiques, seule l'activité biologique, en particulier celle des Lombricides, est responsable de la migration des pollens.

Dans le marais de Dol les dépôts tourbeux sont peu épais et recouverts par 0,50 à $2,20 \mathrm{~m}$ de sables et tangues dépourvus de pollens autres que ceux des Chénopodiacées et de Pinus qui sont contemporains de la sédimentation. L'infiltration des pollens se manifeste, sous la tourbe, à la partie supérieure de la tangue sur une dizaine de centimètres.

$c$ - Les risques de contamination par remaniement de matériel organique ancien sont importants dans les sédiments marins et fluviatiles. Si le sédiment remanié est antéquaternaire, comme c'est le cas, pour parler d'un problème que nous connaissons bien, dans les paléovallées de la Manche, la distinction entre pollen remanié et pollen contemporain sera aisée (M.T. Morzadec et G. Delibrias, 1972). Par contre elle sera impossible si le sédiment remanié est quaternaire.

En général ce type de contamination n'affecte pas les dépôts tourbeux et ce sont eux que l'on soumet le plus souvent à l'épreuve du radiocarbone. Il ne devrait donc pas intervenir dans notre analyse bien qu'il demeure possible car les tourbes sont ici littorales.

\section{3) Remarques}

Ainsi des raisons fort variées peuvent contribuer à expliquer les désaccords observés. Cependant le transport de pollens d'une couche tourbeuse à l'autre est, on l'a vu, assez peu probable ; de plus ici les lentilles tourbeuses sont bien en place. Il ne s'agit pas de résidus de tourbières arrachés par la mer car chaque niveau présente des caractères propres et on suit l'évolution de la végétation herbacée en fonction des oscillations du niveau marin: évolution d'un marais à Cypéracées, lacustre, vers un schorre à Chénopodiacées par exemple lors d'une oscillation positive. Il y a donc sans nul doute des contaminations au niveau de la matière organique. A ce sujet, il faut noter que les tourbes du Marais Blanc ne sont pas des tourbes franches mais des tangues tourbeuses et à cet égard que les "bons sondages" sont ceux qui ont été implantés le plus au Sud, dans le marais noir ou en bordure de celui-ci. Or la tangue est de toute évidence un matériel remanié comme en témoigne les dates obtenues pour les échantillons du sondage 9. Qu'en est-il exactement de la matière organique qu'elle contient ? Quelle est la part de carbone qui revient à la matière organique contemporaine du dépôt, à la tangue plus ancienne ou à l'humus récent entraîné par les eaux ? Selon l'importance relative de ces deux derniers facteurs on pourrait avoir tantôt un vieillissement tantôt un rajeunissement apparent du dépôt.

Cependant avant de retenir cette explication comme valable, il faut examiner si de telles pollutions sont quantitativement vraisemblables. Ainsi pour rajeunir un échantillon de 5850 ans jusqu'à un âge apparent de 4250 ans (ce qui serait le cas du sondage 9), il faut admettre un apport de $20 \%$ de carbone récent. Il s'agit alors non plus d'une pollution, mais d'un mélange : ce qui rend l'éxplication difficilement acceptable. Il n'en reste pas moins. vrai que le pré-traitement, l'élimination de tous les facteurs de contamination est l'un des problèmes les plus importants lorsqu'on fait appel pour les datations radiocarbone à un matériel non homogène, aussi spécial que les tangues tourbeuses.

\section{VARIATIONS DE LA LIGNE DE RIVAGE (fig. 5)}

A la suite de l'étude de ces sondages il est possible de retracer l'évolution de la ligne de rivage dans le fond de la baie du Mont-Saint-Michel mais par contre impossible de tracer une courbe précise des variations du niveau marin. Il est en effet très difficile de tenir compte de façon rigoureuse des profondeurs obtenues pour chacune des lentilles tourbeuses, d'une part à cause du tassement différentiel des sédiments du ma- 


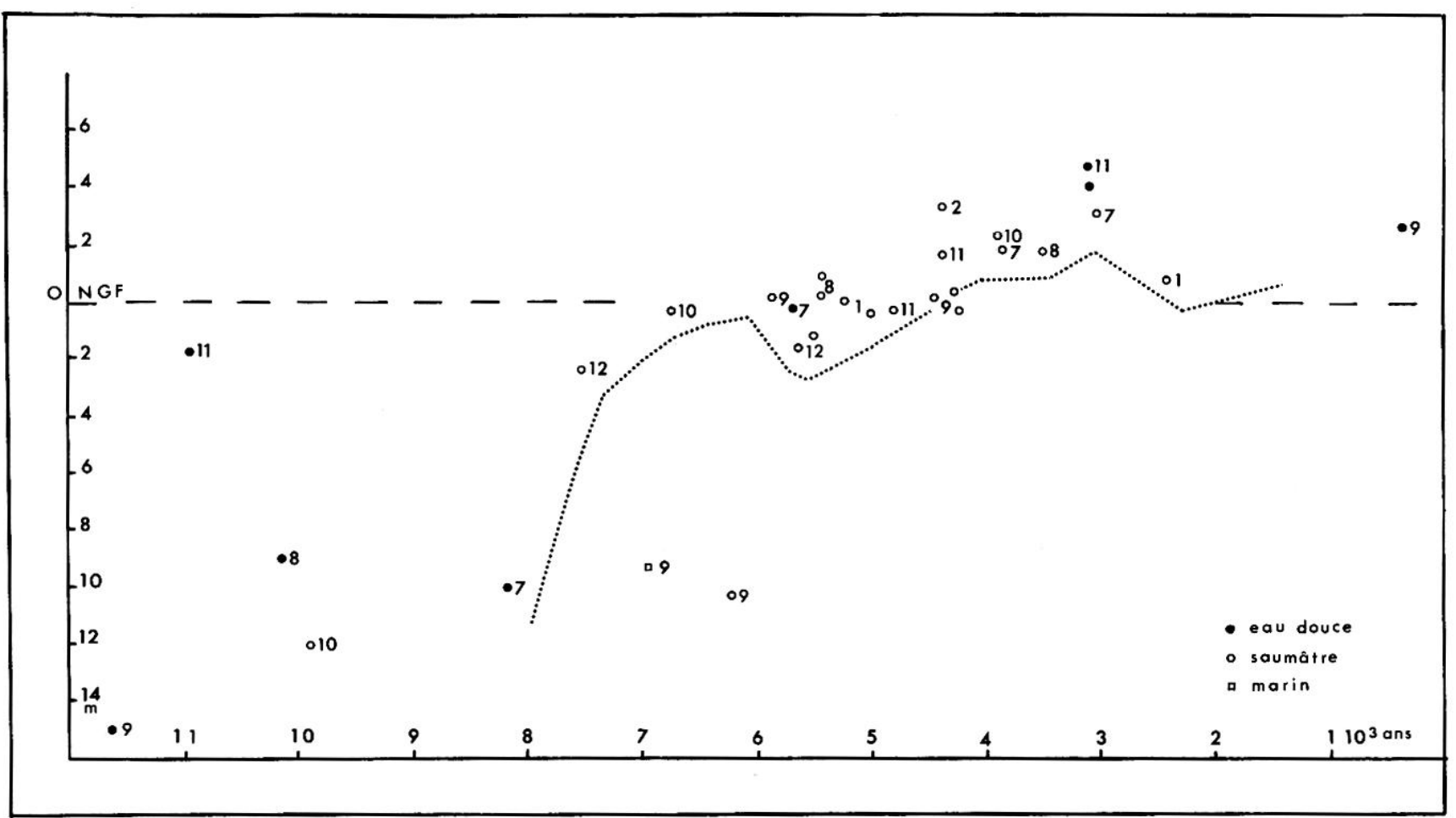

Fig. 5. - Oscillations du niveau des plus hautes mers dans le fond de la baie de Saint-Michel, au Flandrien. Le tassement important des sédiments ne permet pas de tracer une courbe de variation du niveau marin, cependant trois tendances régressives se manifestent par la formation de niveaux tourbeux.

rais : tassement responsable de la dénivellation existant entre les marais noirs tourbeux ( +3 m N.G.G.) et les marais blancs au sous-sol de tangue essenticllement (+6 m N.G.F.), d'autre part à cause du tassement de la tourbe dans le carottier qui rend la cote des prélèvements assez incertaine dans beaucoup de cas. On peut affecter les niveaux d'un certain coefficient d'incertitude mais celui-ci est difficile à calculer, il varie selon la position géographique et selon la profondeur de la lentille tourbeuse. Seuls les échantillons de la base (argile grise de base) n'ont subi aucun tassement.

La mer atteint le fond de la baie du Mont-SaintMichel à la fin du Boréal (vers 8200 ans). Au cours de l'Atlantique, elle l'envahit largement jusqu'au pied de la falaise fossile. Dès la fin de l'Atlantique le rythme de la transgression se ralentit et le fond de la baie apparaît tantôt dans le domaine intertidal tantôt dans le domaine continental. La formation des lentilles tourbeuses se situe à la fin de l'Atlantique - début du Subboréal, entre 5850 et 5400 ans, au Subboréal, entre 3900 et 3450 ans, et au Subatlantique à partir de 3000 ans.

L'évolution de ces marais se fait à partir d'un schorre à Chénopodiacées, vers un marais plus lacustre à Graminées et Cypéracées; au sommet le retour aux conditions halophiles se marque par un développement des Chénopodiacées. Leur formation peut être expliquée par une montée irrégulière du niveau marin. Les tourbes correspondent à un stationnement ou à un léger recul de la mer; les tangues marines qui les surmontent représentent, par contre, une accélération du mouvement général de la transgression. Ceci se traduit sur la courbe de remontée du niveau de la mer que l'on obtient en tenant compte de la position des niveaux saumâtres, par de légères oscillations. Cette courbe s'apparente à celles obtenues pour le N.W. de l'Europe par H. Godwin 1943, N.A. Mörner 1969, J.T. Greensmith et E.V. Tucker 1973, L.P. Louwe Kooijmans 1974; étant bien entendu que pour la courbe du marais de Dol l'altitude ne peut être prise en considération.

\section{BIBLIOGRAPHIE}

Bastin B., 1971. - Recherches sur l'évolution du peuplement végétal en Belgique durant la glaciation de Würm. Acta Geographica Lovaniensia, vol.9, 136 p. Louvain.

Bourcart J. et Boillot G., 1960. - La répartition des sédiments dans la baie du Mont-Saint-Michel. Rev. Géogr. Phys. et Géol. Dyn., vol. III, p. 189-199. Paris.

Desdoigts J.Y., 1970. - Le Marais de Dol. Etude Géomorphologique. Mém. Labo. Géomorph., $\mathrm{n}^{\circ} 18$, 236 p. Dinard. 
Dimbleby G.W., 1962. - The development of British heathlands and their soils. Oxford Forestry $\mathrm{Me}$ moirs, $\mathrm{n}^{\circ} 23,120 \mathrm{p}$. Oxford.

Dubois G., 1924. - Recherches sur les terrains quaternaires du Nord de la France. Mém. Soc. géol. Nord, vol. VIII, 357 p. Lille.

Godwin H., 1943. - Coastal peat-beds of the British Isles and North Sea. J. Ecol., 31, p. 199-247.

Greensmith J.T. and Tucker E.V., 1973. - Holocene transgressions and regressions on the Essex Coast outer Thames estuary. Geol. en Mijnb., vol. 52, p. 193-202. Leiden.

Lebesconte P., 1898. - Périodes géologiques galloromaine et franque. Leurs relations avec le Quaternaire, le Pliocène et l'époque moderne. Bull. Soc. Sci. medic. Ouest, p. 354408. Nantes.

Louwe Kooijmans L.P., 1974. - The Rhine/Meuse delta. Four studies on its prehistoric occupation and Holocene Geology. Analecta Praehistorica leidensia, VII, 421 p. Leiden.

Manet, Abbé F., 1829. - De l'état ancien et de l'état actuel de la baie du Mont-Saint-Michel et de Cancale. 183 p. Saint-Malo.

Mörner N.A., 1969. - Eustatic and climatic changes during the last 15000 Years. Geol. en Mijnb., vol. 48 , p. 389-399. Leiden.
Morzadec-Kerfourn M.T. et Délibrias G., 1972. - Analyses polliniques et datations Radiocarbone des sédiments quaternaires prélevés en Manche centrale et orientale. Mém. B.R.G.M., n 79, p. 160-165.

Morzadec-Kerfoum M.T., 1974. - Variations de la ligne de rivage armoricaine au Quaternaire. Analyses polliniques de dépôts organiques littoraux. Mém. Soc. Géol. Min. Bretagne, $\mathrm{n}^{\circ}$ 17, 208 p. Rennes.

Munaut A.V., 1967. - Recherches paléo-écologiques en Basse et Moyenne Belgique. Acta Geographica Lovaniensia, vol. 6, 191 p. Louvain.

Musset R., 1922. - The geographical characteristics of the Western France. Geogr. Rev., p. 84-89.

Olsson I. U., 1972. - A critical analysis of ${ }^{14} \mathrm{C}$ datings of deposits containing little carbon. Proceedings of the 8 th International Conference on Radiocarbon dating, Wellington, New Zealand, Oct. 1972.

Sirodot S., 1878. - Age du gisement du Mont Dol. Constitution et mode de formation de la basse plaine dite marais de Dol. C.R. Acad. Sci., LXXXVII, p. 267-269.

Sirodot S., 1891. -- De l'âge relatif du gisement quaternaire du Mont Dol. C.R. Acad. Sci., CXII, p. $1180-1182$.

Verger F., 1968. - Marais et Wadden du littoral français. 544 p. Biscaye Ed. Bordeaux. 